\title{
Review
}

\section{The role of real time three-dimensional transoesophageal echocardiography in acquired mitral valve disease}

\author{
Agnè Drasutienė ${ }^{*}$ Sigita Aidietienè, Diana Zakarkaitè \\ Centre of Cardiology and Angiology, Vilnius University Hospital Santariškiu Klinikos, Vilnius, Lithuania \\ Received 21 August 2015; accepted 2 November 2015
}

\begin{abstract}
Summary
Real time (RT) three-dimensional (3D) imaging is one of the most significant developments of the last decade and is now being used with increasing frequency in echocardiography and interventional cardiology laboratories. Improvements in transducer technologies and the development of a matrix array transducer were the most important achievements that lead to the new era of real time 3D transoesophageal echocardiography (TEE). RT 3D TEE is ideal for assessing the mitral valve (MV) because of its high spatial resolution altering in improved MV anatomic detail. This imaging modality provides images of high quality which is of a great clinical value in making diagnosis of MV disease and a valuable tool for surgeons and interventional cardiologists in planning and guiding interventional procedures. This review is intended to provide data about normal MV anatomy and clinical usefulness of RT 3D TEE in defining acquired MV pathology.
\end{abstract}

Seminars in Cardiovascular Medicine 2015; 21:16-26

Keywords: transoesophageal echocardiography, mitral valve

\section{Introduction}

Echocardiography is the most important technique to confirm the diagnosis of valvular heart disease, as well as to assess its severity and prognosis. Transoesophageal echocardiography (TEE) in case of mitral valve (MV) disease should be considered when transthoracic examination is of suboptimal quality or when thrombosis, prosthetic dysfunction or endocarditis is suspected. It should be performed intraoperatively to monitor the results of valve repair or complex procedures [1].

3D echocardiography is an established technique which shows valvular morphology with real-time dynamics in the beating heart. In 2007, the real-time three-dimensional (RT 3D) TEE fully sampled matrix array transducer (X7-2t, 2-7 MHz, 2500 piezocrystals - Philips Medical Systems) introduced TEE 3D modality in addition to usual two-dimensional (2D) imaging [2-4]. RT $3 \mathrm{D}$ TEE is ideal for assessing the MV because

\footnotetext{
* Corresponding address: Agnè Drąsutienè, Centre of Cardiology and Angiology, Vilnius University Hospital Santariškiu Klinikos, Santariškių 2, Vilnius, Lithuania.

E-mail: agne.drasutiene@santa.lt (A. Drasutienè).
}

of the perpendicular position of the ultrasound beam to the MV and the close proximity of the TEE transducer to the valve. Also, 3D TEE has high spatial resolution, resulting in improved MV anatomic detail. Therefore, assessments of native and prosthetic MV pathology are among the top indications for a 3D TEE examination [5].

Recently suggested clinical applications of 3D TEE in evaluation of the mitral apparatus are: defining the extent and location of pathology, determining the mechanism and severity of valvular dysfunction and, when appropriate, communicating the results of the echocardiographic examination to the interventional cardiologist or cardiac surgeon when an intervention is required [6].

\section{Data acquisition and post-processing}

A systematic approach performing a 3D TEE mitral valve examination is recommended. A systematic 2D TEE examination includes 4-chamber, 2-chamber, and long-axis views from the midesophageal position, as well as short-axis and long-axis views from the transgastric position. Usually not all views can be optimally obtained 
in different patients and additional views may be required to obtain relevant information in patients with complex valve pathology. Initially, a real-time 3D TEE and a subsequent gated 3D data set should be acquired up to 120 mid-esophageal views with and without color (zoomed acquisition) to determine the whole valvular structure [6].

The recently available 3D matrix array transducers and echo systems have RT 3D volume imaging available in the following modes: live 3D narrow volume, live 3D zoomed, live 3D full volume and live 3D color Doppler [2].

\section{Real-time live $3 D$}

A pyramidal set of $50 \times 30^{\circ}$ is displayed in real time. The $3 \mathrm{D}$ image changes as the transducer is moved. In practice, it is very useful for capturing an image of the aortic valve in its entire volume. Other structures (e.g., mitral valve, atrial septum, right atrium structures) are visualized only partially, because of the narrow volume data set. One of the advantages of live 3D modality is the relatively high frame rate $(20-40 \mathrm{~Hz})$.

\section{Live 3D zoom modality}

This modality can display a magnified pyramidal data set. After sizing the zoom sector over the region of interest in 2D TEE, the button of 3D zoom modality is switched on and RT 3D view is displayed. Minimizing sector width is important for increasing temporal resolution and image quality. This mode provides good quality images of the MV, the left atrial appendage, and the atrial septum. Both live and zoom modalities are devoid of artifacts commonly encountered with ECG gated 3D acquisition.

\section{Live 3D full volume}

A pyramidal volume of $60 \times 60^{\circ}$ up to $100 \times$ $100^{\circ}$ allows the inclusion of large cardiac structures. The full volume is constructed from narrow volume data from 2 to 7 heart cycles which are stitched together to form a single pyramidal set. This modality requires ECG gating. Accordingly, it may suffer from artifacts due to the incorrect position of the narrow volume data. Acquiring the data when the patient holds his breath can minimize such artifacts. In this modality the temporal resolution is higher when compared with zoom modality (up to $40 \mathrm{~Hz}$ ). However, spatial resolution (particularly lateral resolution) deteriorates compared with RT acquisition.

\section{D full volume color Doppler}

Similar to the full volume method described, this modality is constructed by merging 7-14 narrow pyramidal sets. Although larger data volumes are achieved with multiple-beat full-volume color Doppler acquisition, it is limited by stitching artifacts. The final representation, however, is a narrow pyramid, since two different modalities must be acquired - the anatomy and the flow. Thus it is essential to put the flow jet of interest in the center of the sector. 3D TEE acquisition currently provides significantly better color Doppler image quality compared to TTE and therefore is recommended for detailed color flow analysis [1].

As mentioned above, a data set acquired per single heart beat, overcomes such limitations as breathing or rhythm disturbances, but has lower spatial and temporal resolution. While images acquired with multiple-beat 3D echocardiography are of higher temporal resolution, still they are prone to imaging artifacts created by inspiration/expiration motion or irregular cardiac rhythms. Finally, once volumetric data are obtained, one can rotate, angulate and crop images to assure immediate, en-face view of MV similar to that seen by surgeons (Fig. 1).

\section{D optimization}

Low gain settings result in echo dropout, with the potential of artificially eliminating anatomic structures that cannot be recovered during postprocessing. Alternatively, with excess gain, there is a decrease in resolution and a loss of the $3 \mathrm{D}$ perspective or depth within the data set (Fig. 2). As a general rule, both gain and compression settings should be set in the midrange (50 units) and optimized with slightly higher time gain (time gain compensation) to enable the greatest flexibility with post-processing gain and compression [6].

\section{Normal mitral valve anatomy}

The normal MV consists of the annulus, valvular leaflets and subvalvular apparatus composed of a highly variable chordae tendineae arrangement with dual papillary muscles.

\section{MV annulus}

From an anatomical point of view mitral annulus is a structure supporting both mitral leaflets. The mitral annulus is an incomplete connective tissue ring that has an elliptical or saddle-shaped configuration. The longest diameter runs from commissure to commissure, whereas the shortest one runs from the middle point of the anterior segment to the middle point of the posterior segment of the annulus. MV annular geometry can be evaluated using 3D TEE by the use of serial cropping of the large-sector data set until the circumference of the annulus is found. For a true 


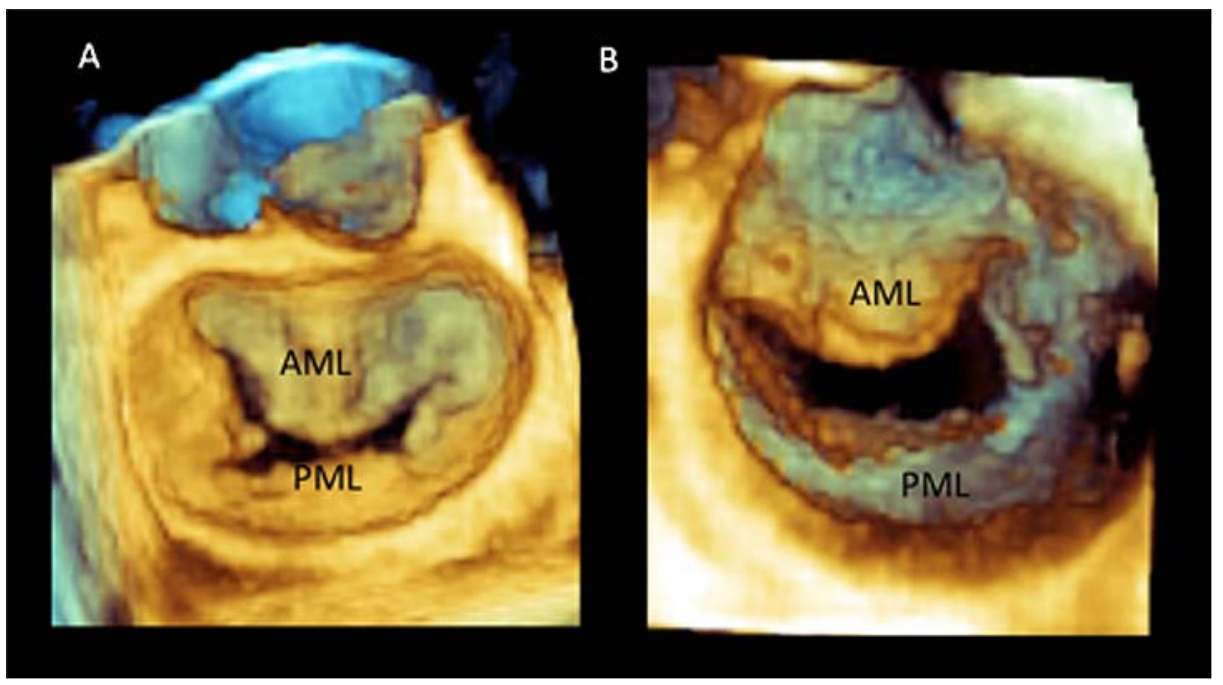

Figure 1. 3D presentation of mitral valve - "en-face" view: mitral valve displayed centrally with the aortic valve placed superiorly, regardless if the valve is oriented as viewed form atrium (A) or from left ventricle (B). AML - anterior mitral leaflet, PML posterior mitral leaflet.

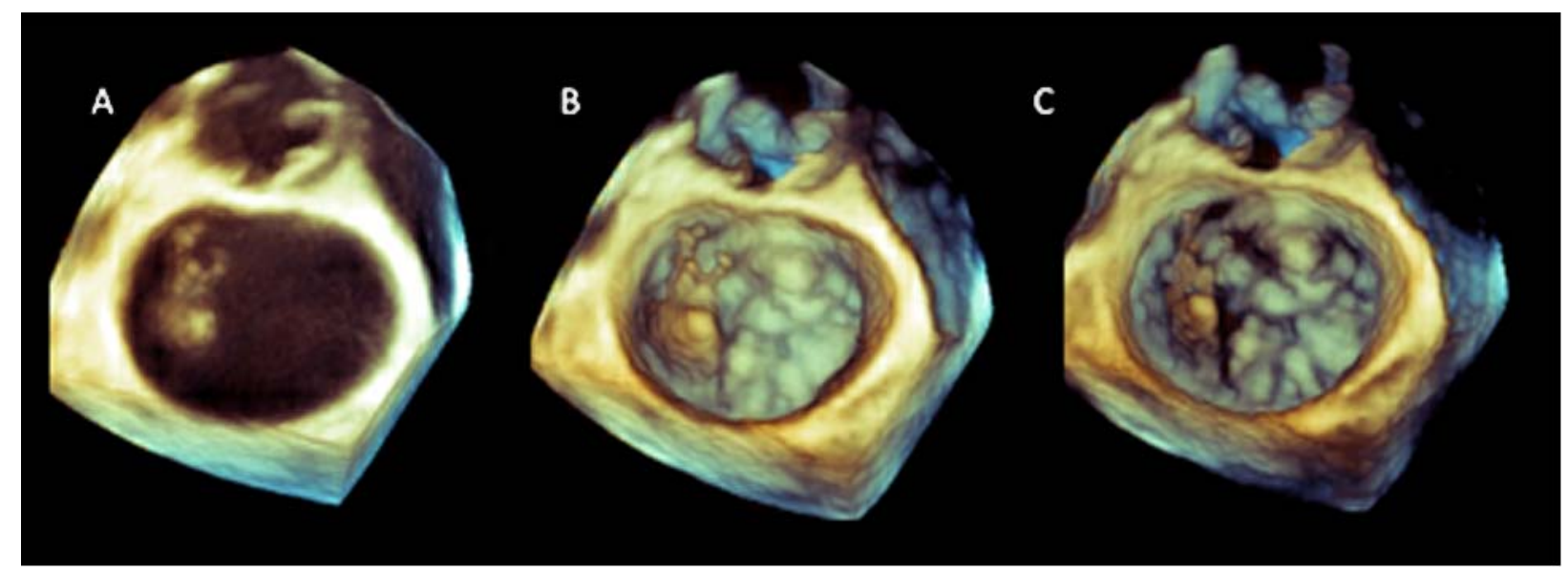

Figure 2. Gain settings: (A) An excess of gain (70) decreases image resolution and depth perspective; (B) - normal gain (40) settings; (C) too low gain (10) results in echo dropout artefacts.

3D rendition of the MV annulus 3D offline processing is required. 3D modeling software is used to trace the contour of the MV leaflets and annulus, with resultant representative images [7]. Through these reconstructions, it is now possible to measure mitral annulus diameters, area, circumference, segmental and overall volume of the leaflets and the aortic-mitral plane angle (Fig. 3).

\section{MV leaflets}

The normal mitral valve has two leaflets that are conventionally described as anterior and posterior leaflets. Because of the elliptical mitral annulus shape, both mitral leaflets are not equal in size [8]. The anterior (aortic) leaflet has a triangular-shaped free edge, attaches to one-third of the annulus and has a wider surface with a shorter base. The narrower posterior (mural) leaflet covers two-thirds of the annular circumference. The surface area of both leaflets taken together is 2.5 times the area of the valvular orifice [9]. The anterior leaflet does not attach to the annulus fibrosus, but has fibrous continuity with the interleaflet triangle underneath the two aortic leaflets - left and non-coronary (Fig. 4). Slits in the posterior leaflet create three scallops which, following Carpentier's classification [10], are labeled from lateral to medial as P1, P2, P3. The corresponding areas of the anterior leaflet, although the anterior mitral leaflet is not anatomically divided, are termed A1, A2, A3. In some cases, particularly when the valve tissue is oversized, Duran's classification [11] is appropriate. It divides the valve into anterolateral and posteromedial halves. The middle cusp of the posterior leaflet is divided further into anterolateral (PM1) and posteromedial sections (PM2), Fig. 5.

By properly cropping the volume data set of $\mathrm{MV}$, sections comparable to that of 2D TEE can be 


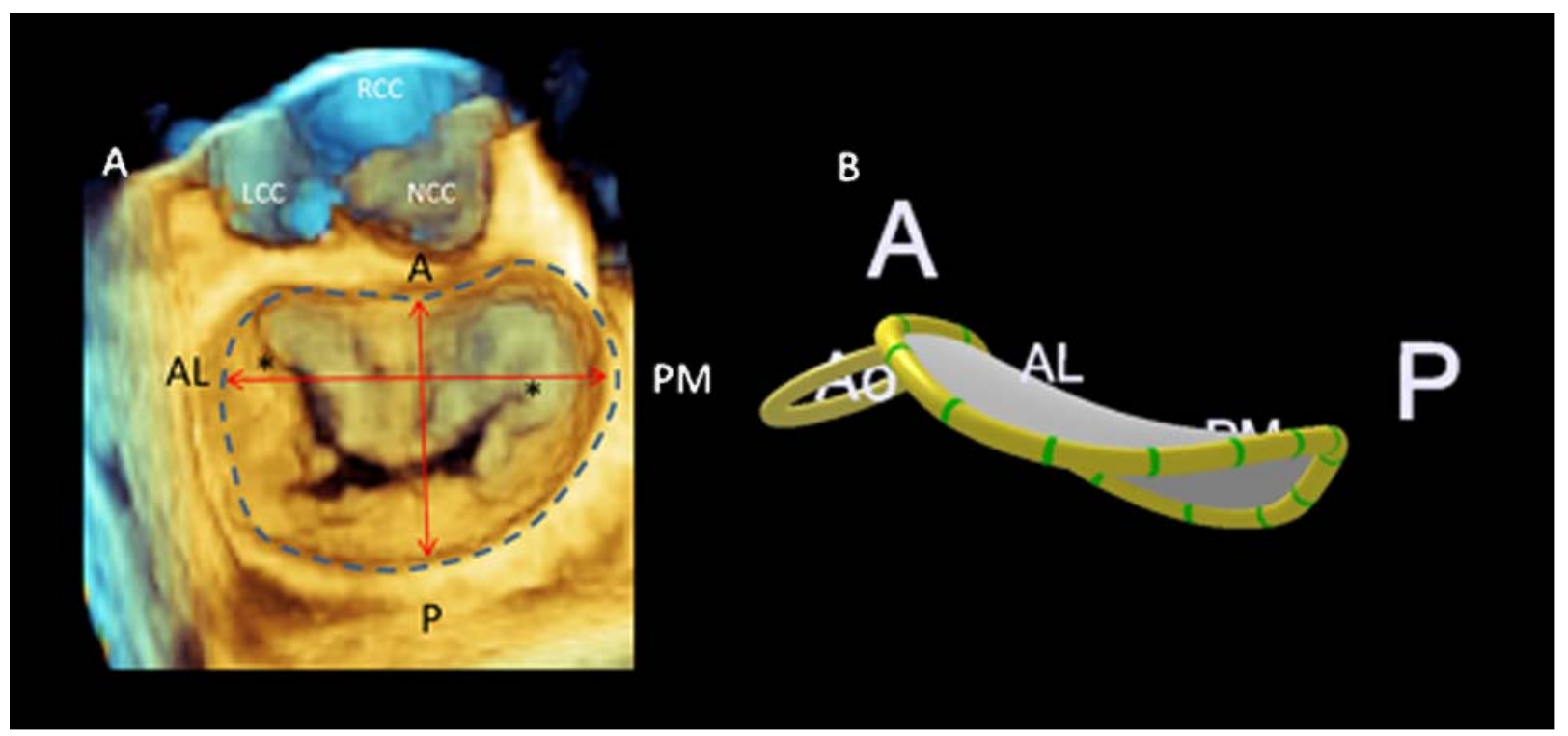

Figure 3. (A) Anatomical view of mitral valve. Annulus is D-shaped, in which the longest diameter is anterolateral-posteromedial (AL-PM) and the shortest diameter is antero-posterior (A-P). Asterisks show both the anterolateral and posteromedial commissures. (B) MVQ (mitral valve quantification) plugin for QLAB software offers a semi-automated analysis package for accurate measurements of the mitral annulus, valve commissures, leaflet coaptation and topography and relative location of the aortic valve.

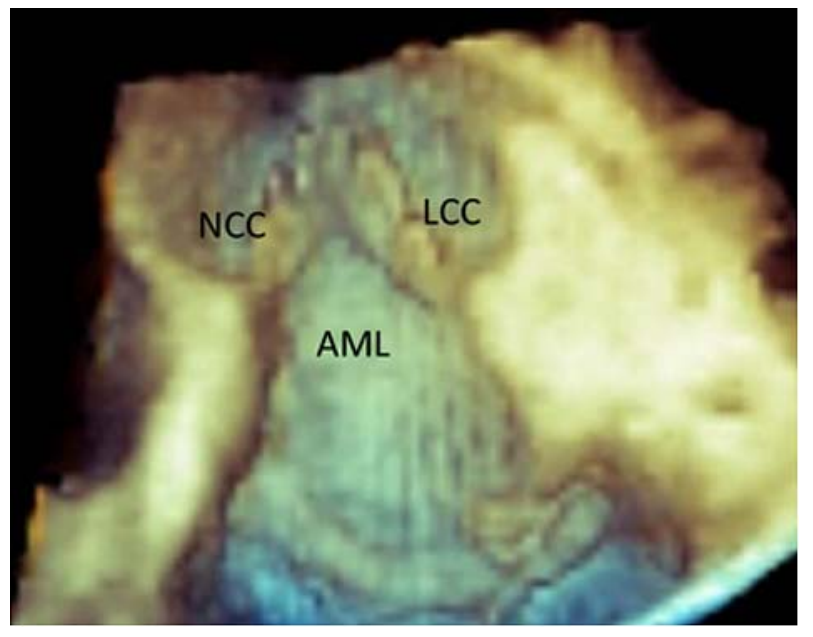

Figure 4. The interleaflet triangle and aorto-mitral continuity from LV perspective. NCC - non-coronary cusp, LCC - left coronary cusp, AML - anterior mitral leaflet.

obtained, making real-time 3D TEE an intuitively educational tool (Figs. 6 to 8 ).

The anterior and posterior leaflets are separated by two commissures - anterolateral and posteromedial. The anterolateral commissure is next to the left fibrous trigone and the left atrial appendage, while the posteromedial commissure is next to the right fibrous trigone and the interatrial septum. The commissures are the morphologic regions of the valve that do not extend completely to the annulus, thus the leaflet tissue forms a continuous ring around the annulus (Fig. 9).

\section{Subvalvular apparatus}

The MV leaflets are supported by chordae, that originate in the papillary muscles (lateral wall of the left ventricle), bifurcate several times, and attach to the ventricular aspects of both leaflets [12]. Unlike the anterolateral papillary muscle, the posteromedial muscle has a single blood supply and thus is more vulnerable to ischemia and rupture. Importantly, mitral geometry and function are influenced by the geometry and function of both the left atrium and ventricle.

The chordal attachments and the tips of the papillary muscles can be evaluated with a live 3D full volume - pyramidal data set. However, the best view of the whole subvalvular apparatus is provided by a transgastric long axis view, with both MV and left ventricle included [7].

\section{Mitral regurgitation}

Mitral regurgitation (MR) is an important cause of morbidity and mortality in developed countries $[13,14]$. Major causes of MR are organic valve disease (degenerative or rheumatic MR) and secondary changes in MV apparatus due to dilated or ischemic cardiomyopathy (functional MR). Degenerative $\mathrm{MR}$ is a result of myxomatous degeneration or fibroelastic deficiency (FED) of MV tissue. It is the most common MR etiology in Europe [14].

A unique advantage of real-time 3D TEE is that it provides a comprehensive picture of the entire MV in one shot, simulating the surgical view of the valve. Furthermore, flail or prolapsed leaflets 


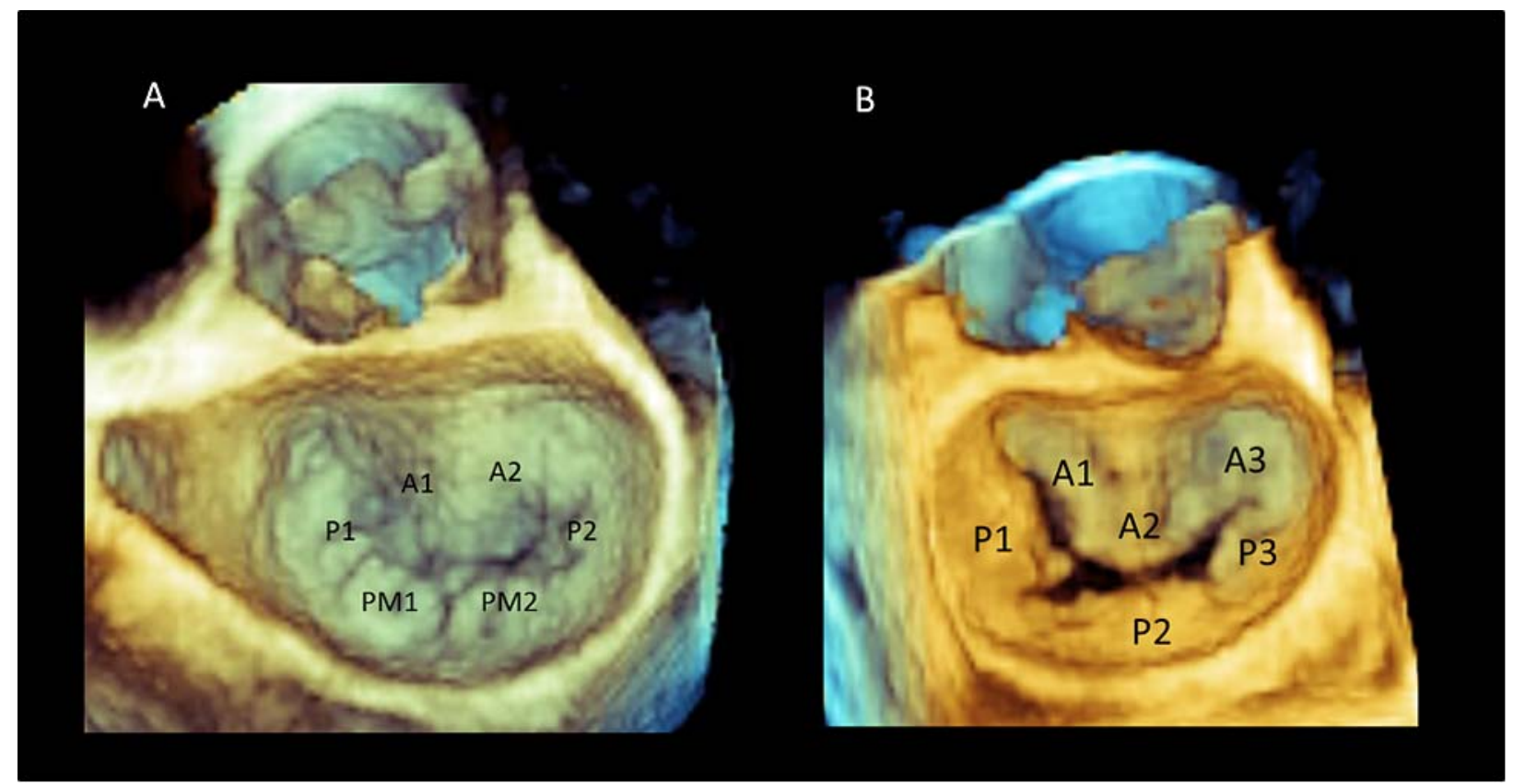

Figure 5. (A) Multiple scallops of posterior leaflet. Duran classification divides anterior leaflet into two scallops - A1 and A2, and posterior leaflet into P1, PM (medium) which has two scallops PM1 and PM2, and most laterally - P2; (B) According to Carpentier classification both mitral leaflets are divided into three scallops.

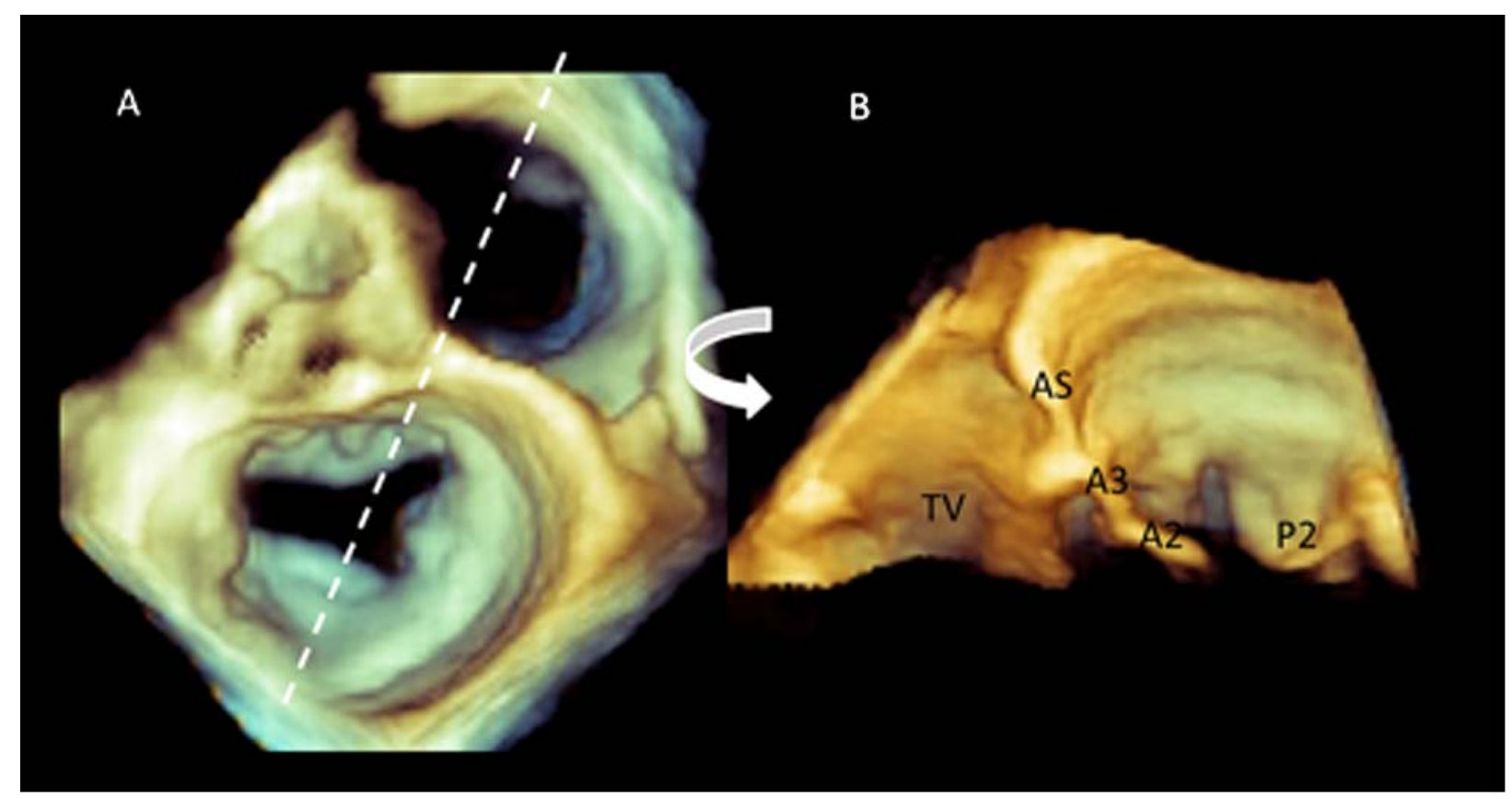

Figure 6. 3D TEE imaging of mitral valve, sections comparable to that of $2 \mathrm{D}$ TEE can be visualized by cropping and rotating data set. Cutting plane cuts the atrial septum (dotted line) perpendicularly and obtains the 4-chamber. In this section A3, A2 and P2 scallops are visualized. AS - atrial septum. TV - tricuspid valve.

imaged from this perspective can be seen in motion.

Chandra et al. demonstrated the feasibility of using RT 3D TEE-derived volumetric measurements of valvular anatomy to objectively evaluate MV annular and leaflet distortion in patients with degenerative mitral valve disease before surgery. Furthermore, they found that morpho- logical analysis of MV is able to differentiate between degenerative and normal valves, and, more importantly, between the two different etiologies of degenerative MV disease, namely Barlow disease (myxomatous degeneration) and FED [15].

Mitral valve with myxomatous degeneration has complex pathology and consists of a di- 


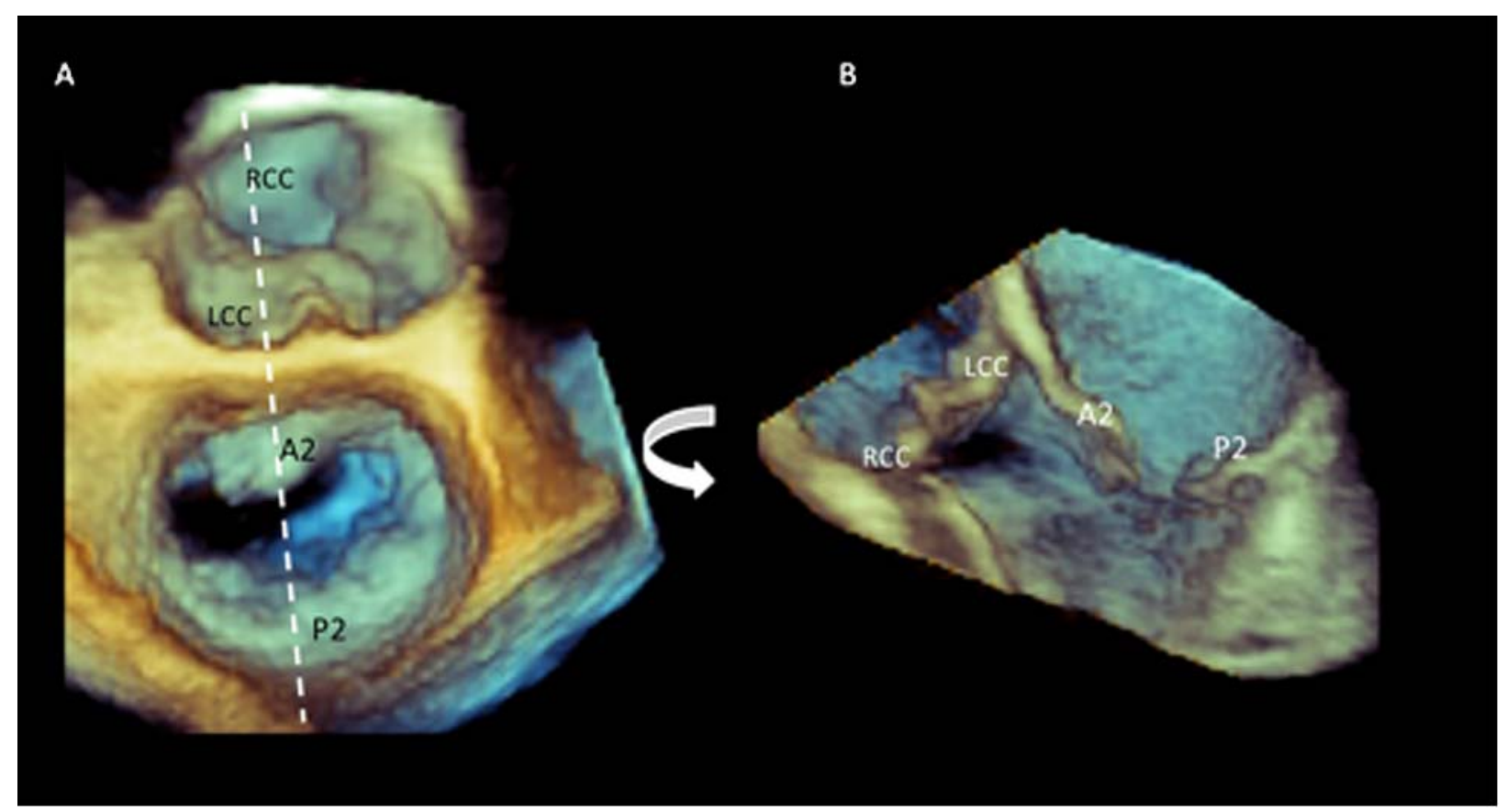

Figure 7. 3D TEE images of mitral valve in full volume modality. Cutting plane (dotted line) passes through the middle part of mitral leaflets and aorta. In this section A2 and P2 scalops are visualized. LCC - left coronary cusp, NCC - non-coronary cusp.

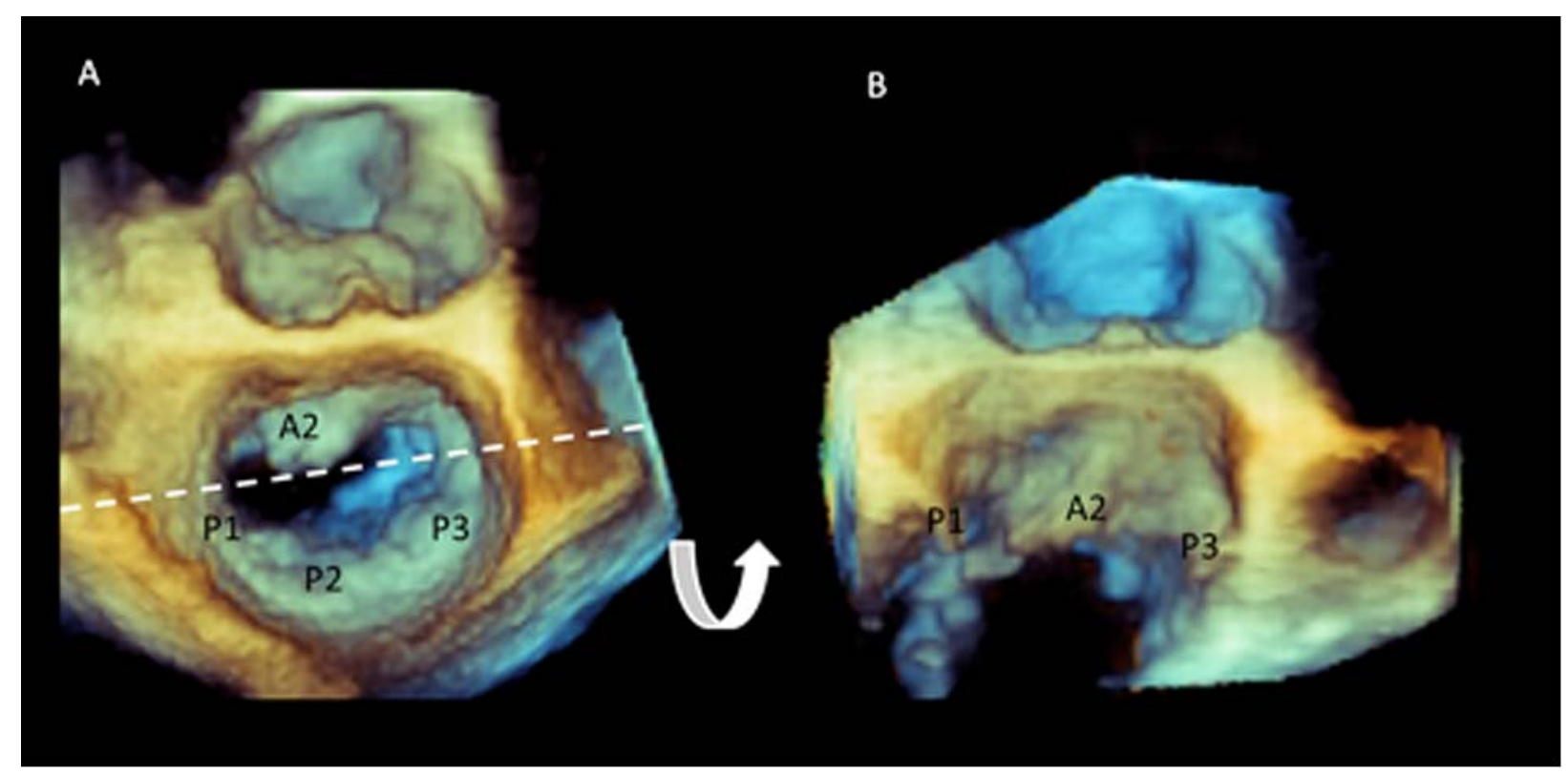

Figure 8. 3D full volume images of mitral valve and cropping line (dotted) passing on the two commissures (A). Rotation of the image obtains 2-chamber view similar to that from 2D TEE (B). In this section P1, A2 and P3 scallops are visualized.

lated annulus, thick leaflets, excess tissue, chordal elongation with multisegmental billowing and prolapse of leaflets. In contrast, FED has a less complex pathology with thin, flail chordae, mild annular dilation, and transparent leaflets with involvement of only one segment, usually frequently an isolated P2 prolapse as illustrated in Figs. 10-11 [16]. The information gained from TEE 3D echocardiography has especially made an impact in guiding clinical decisions in the evaluation of MV disease. The decision of early surgery in degenerative MV disease is based on the suitability of repair, and the suitability of repair is generally based on echocardiography. The better understanding of $\mathrm{MV}$ anatomy afforded by 3D echocardiography has been shown to be quite valuable in this setting.

Functional or ischemic mitral regurgitation may develop in the acute or chronic course. The acute MR is secondary to papillary muscle infarction and rupture. In chronic ischemic MR, MV leaks when leaflets and subvalvular appa- 


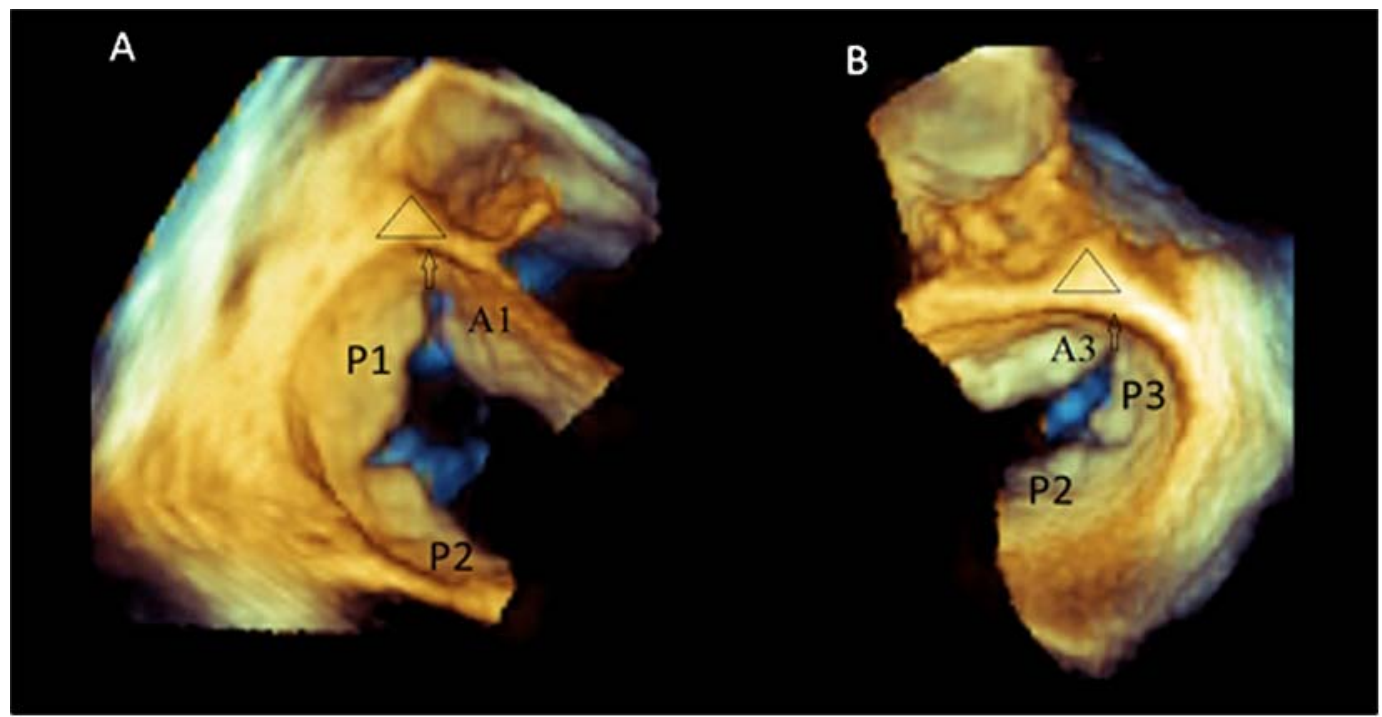

Figure 9. Anterolateral commissure, left fibrous trigone (A), posteromedial commissure and right fibrous trigone (B). Both commissures do not reach the annulus but end 3-5 $\mathrm{mm}$ before the insertion to it.

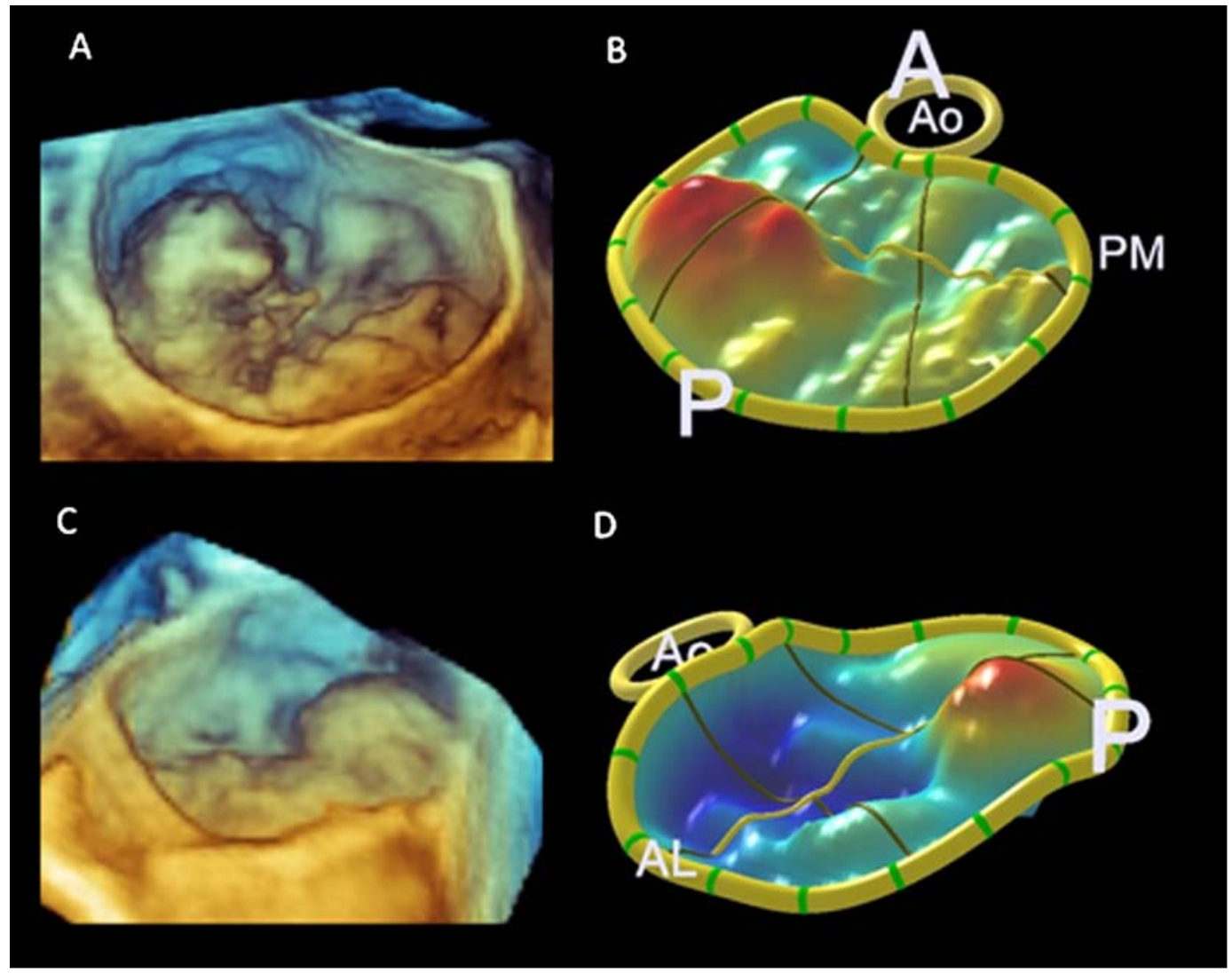

Figure 10. Multisegmental billowed osterior leaflet (Barlow disease) (A) and the corresponding image obtained with MVQ (mitral valve quantification) plugin for QLAB software showing the prolapsing segments of the posterior leaflet protruding above the annular plane (B). Prolapse of the posterior leaflet P3 segment (C) and corresponding MVQ model (D).

ratus appear normal. Chronic MR is therefore not a disease of the valve, but rather a disease of the left ventricle (LV) and is a consequence of a leaflet restriction, which is due to tethering by the chordae and papillary muscles in patients with LV dysfunction, because of the posterior-inferior scar. Usually it appears as an asymmetric deformation of the medial part of MV (accordingly A3/P3 segments). Awareness of this asymmetry might help to avoid underestimation when the proximal isovelocity surface area (PISA) method is used to quantitatively assess the magnitude of mitral regurgitation, illustrated in Fig. 12 [2]. 


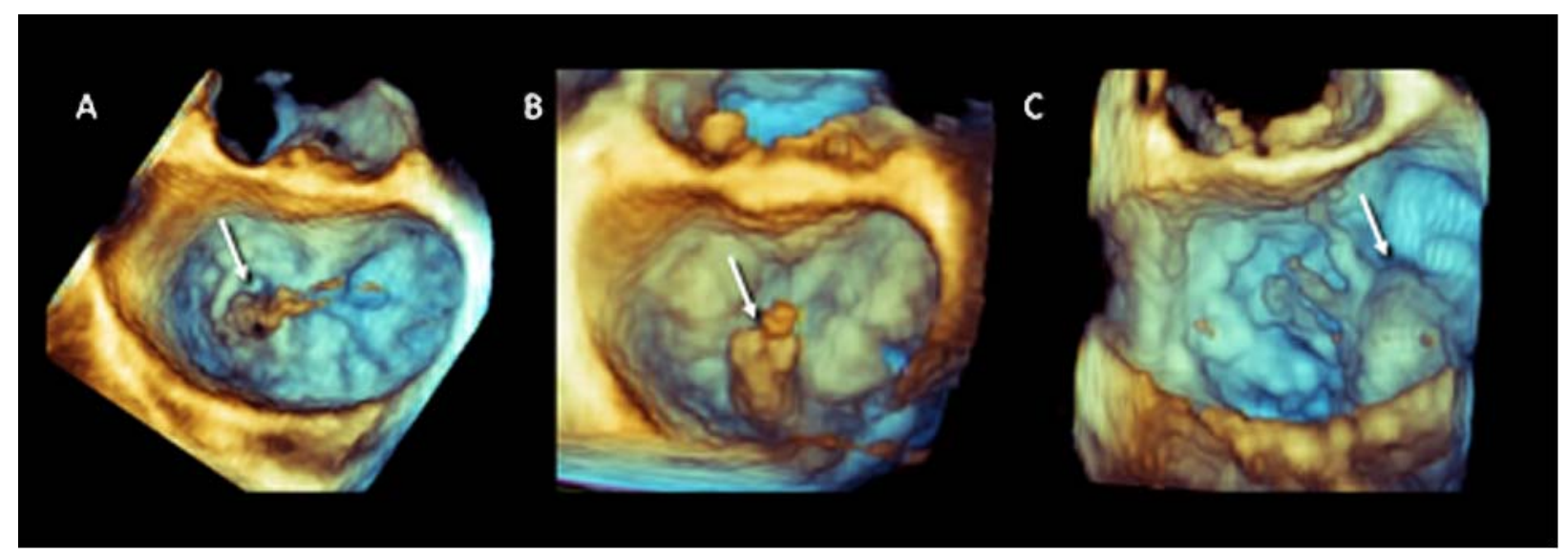

Figure 11. Zoomed 3D TEE view of the mitral valve form the "en-face" view in patients with isolated P1 prolapse (A), fibroelastic deficiency and P2 prolapse with ruptured chorda (B), and Barlow disease with P2 flail segment and three ruptured chordae (C).

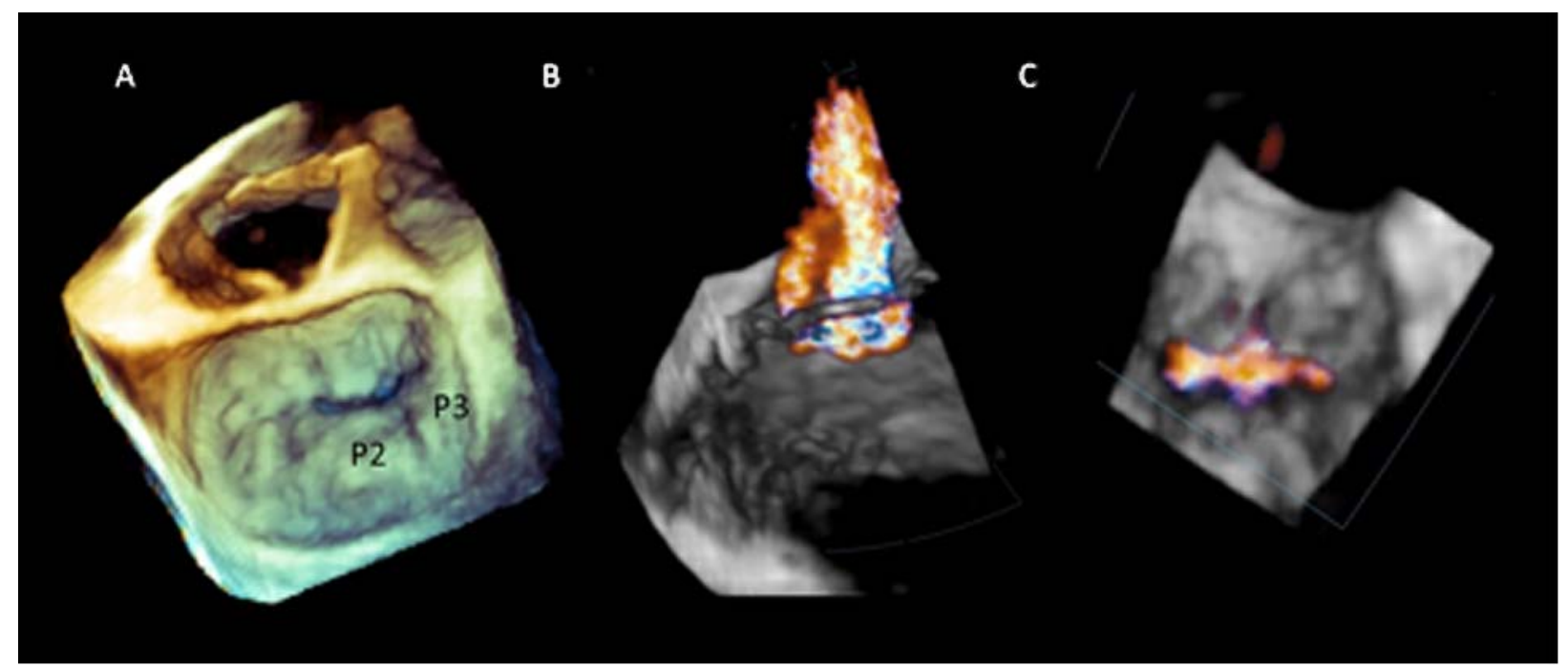

Figure 12. (A) En-face view of the mitral valve. Ischemic mitral regurgitation due to the tethering of posteromedial part of the valve. (B) The proximal isovelocity surface area (PISA); (C) 3D TEE full volume color Doppler image of "vena contracta".

In non-ischemic functional $\mathrm{MR}$, mitral valve leaflets are structurally normal and MR is secondary to the changes in LV geometry resulting from LV dilation, impaired LV function. Thus tethering of the leaflets is symmetric due to annular dilation, providing central regurgitant jet in the region of coaptation defect.

Depending on the complex and mixed mechanisms of functional mitral regurgitation, RT 3D TEE color Doppler may show variable shapes of regurgitant orifices. Ischemic functional MR usually forms an asymmetric regurgitant orifice located near the medial commissure, while a semilunar, symmetric shape in the whole length of coaptation is a cause of nonischemic functional MR, such as dilated cardiomyopathy, global LV remodeling and mitral annular dilation.

\section{Mitral stenosis}

Rheumatic heart disease is overwhelmingly the most frequent cause of mitral stenosis $(99 \%$ of all cases). A chronic rheumatic disease induces chronic scarring and anatomic changes of the MV such as: leaflet thickening, commissural fusion and shortening, thickening and fusion of the chordae. Fibrous bridging across the valvular commissures and calcification create a fish mouth stenosis ("funnel-like") stenotic MV [17]. Therefore, a reliable measurement of the valve area by planimetry requires verification that the chosen plane corresponds to the smallest valve area.

With real-time 3D TEE, the narrowest valve orifice is always imaged, both from above and below the mitral valve (the atrial and ventricular views, respectively). Measurements can be properly made by cropping the volume data set at the level of the image plane that corresponds to the smallest area or using multiplane reconstruction (MPR). The fact that the narrowest valve area is always displayed improves accuracy and reduces interobserver variability, as illustrated in Fig. 13 [18]. 


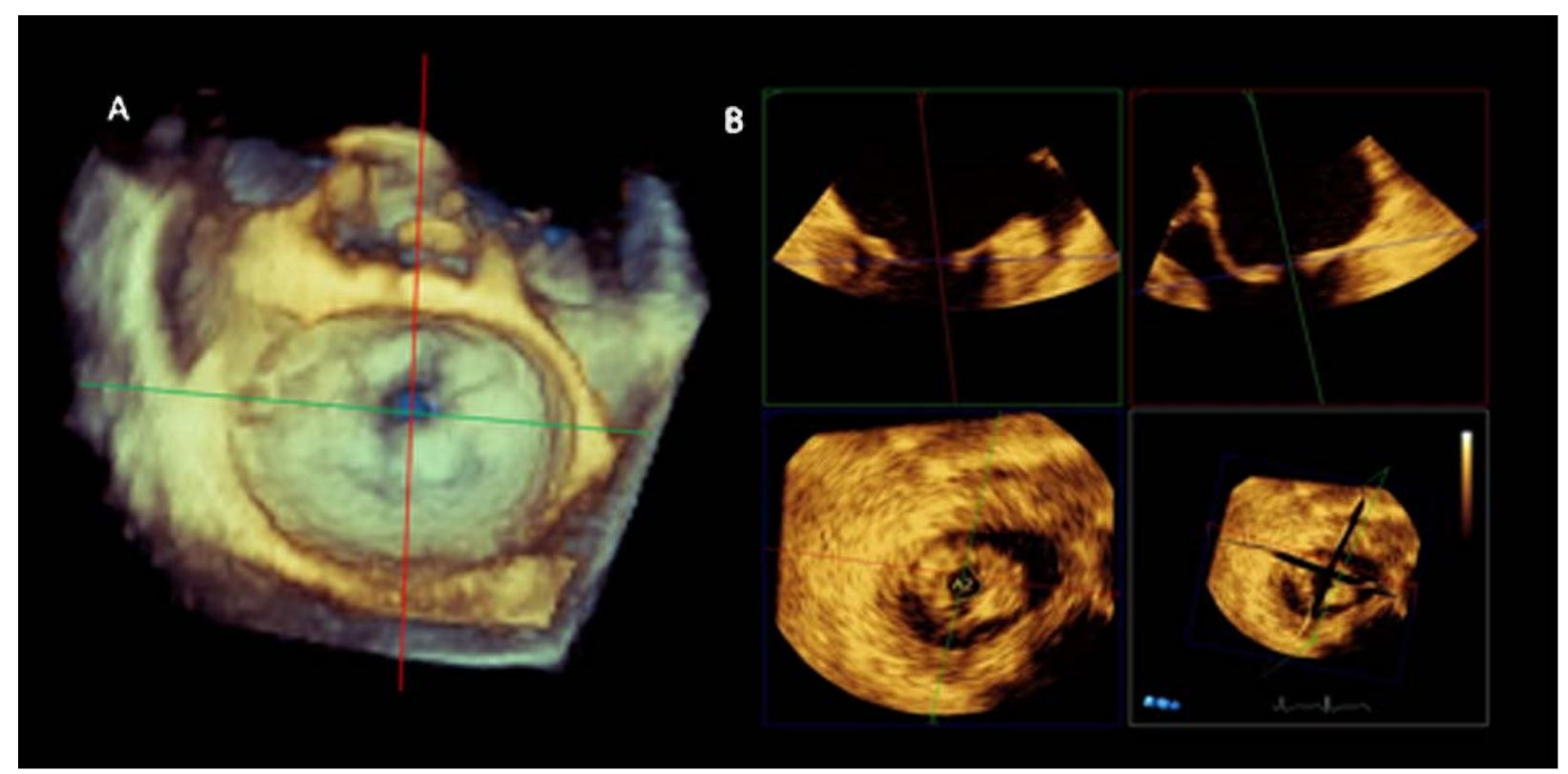

Figure 13. Figure shows stenotic mitral valve and mitral valve area. Using multiplanar reconstruction (MPR), the measurements of the smallest valve area can be properly done by moving cropping platform in three orthogonal planes - red, green and blue (RGB).

In past few years 3D TEE became the most useful technique for evaluating pathological features of the stenotic mitral valve, because the morphology of the MV is considered as the main predictor of a successful percutaneous mitral valve balloon valvuloplasty. Hence the MV scoring system using echocardiography is of crucial importance [19]. Wilkins' scoring system includes leaflet mobility, thickening, calcification and subvalvular evaluation [20]. Osama et al. proposed a new 3DE scoring system [21], still a large-scale trial is needed to improve its application.

A detailed analysis of the MV commissures is of particular interest in patients being considered for MV commissurotomy [22]. The commissures can be best obtained form the mid-oesophageal position by RT live 3D (narrow volume) TEE, which provide us with the best temporal and spatial resolution images. While inspecting the commissures, the fusion and the severity of calcification is observed [11].

Carpentier and colleagues categorize the fused commissures according to three grades of increasing severity [23]. Grade I is a partial fusion of the commissures with preservation of commissural chordae. Grade II is a complete fusion of the commissures with a visible delineation between the anterior and posterior leaflets. Grade III is a complete fusion of two commissures with no visible delineation between both leaflets. Real-time 3D TEE shows clearly whether the fusion is more pronounced in a single commissure (asymmetrical fusion) or conversely, whether both commissures are equally affected (symmetrical fusion) (Fig. 14).

\section{Mitral annular calcification}

Degenerative calcific deposits can develop in the annulus of the MV, visualized as irregular, stony hard, occasionally ulcerated nodules of 2$5 \mathrm{~mm}$ thickness that lie behind the leaflets and are usually located on the posterior annulus. The local calcific deposits generally does not affect the valvular function [17], while ring-shaped calcification may lead to functional mitral stenosis by reducing leaflet excursion without leaflet fusion. The diagnosis of calcified annulus is easily made with 2D images, but extensive calcifications are better recognized in 3D images.

\section{MV treatment and 3D echocardiography value}

MV annulus dimensions obtained using 3D TTE data set correlate well with magnetic resonance imaging (MRI) [24]. Ender and colleagues has been used 3D TEE rendered en face MV image as a virtual model for perioperative sizing of MV annuloplasty ring [25]. To conclude, the off-line construction of a 3D MV model that quantifies annular dimensions and geometry may assist in surgical MV annuloplasty planning.

3D TEE provides optimal prosthetic MV images. The atrial side of the mechanical prosthesis can be visualized perfectly. In contrast ventricular side is hidden by ultrasound artifacts coming from metallic components of the prosthesis. Therefore 3D TEE is of great importance in identifying clot formation on the atrial side of the prosthetic valve [26], presence of paravalvular leak or areas of dehiscence $[27,28]$. The size and location of paravalvular leaks can be quickly 


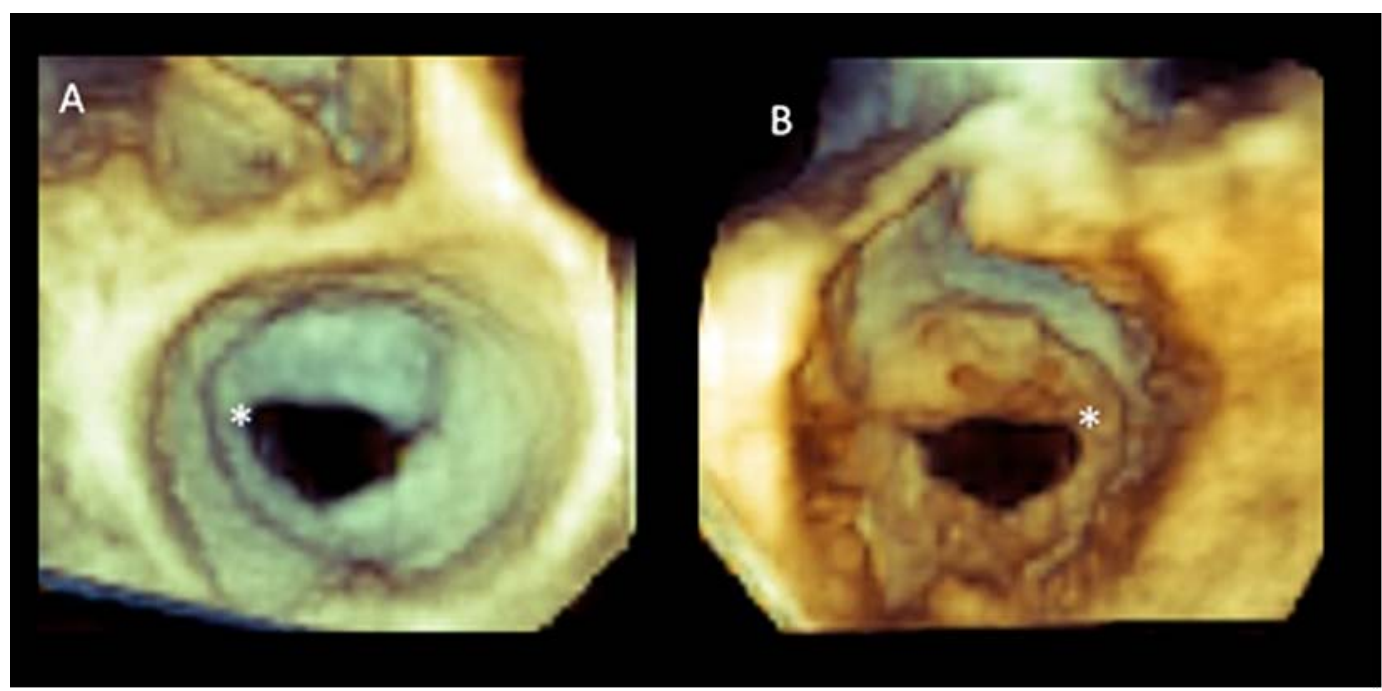

Figure 14. Mitral valve stenosis with asymmetrical commissural fusion both from atrial (A) and left ventricular (B) perspective: anterolateral commissure (yellow asterisk) is completely fused (Carpentier classification grade III) and the posteromedial commissure (black asterisk) is of grade II.

defined using all 3D imaging modes, including 3D color Doppler. Note that low temporal resolution or inadequate gain settings in the zoom and full-volume modes may underestimate or miss a paravalvular gap. The $3 \mathrm{D}$ live mode is also a valuable tool in guiding percutaneous closure of the paravalvular leak [29].

In past decade numerous percutaneous mitral valve repair techniques have been introduced. Real-time 3D TEE is the ideal imaging technique for the guidance of the edge-to-edge repair of mitral valve (mitral clip procedure) [30] or mitral leaflet prolapse repair with neochord implantation on beating heart [31].

In conclusion, real time 3D TEE provides a unique assessment of the mitral valve apparatus, defining the severity and location of valve pathology. The unique aspect of this imaging modality is ability to image the internal valvular structures on the "en-face" view. The ability to visualize long internal structures makes real time 3D TEE suitable tool for monitoring percutaneus mitral valve procedures. Moreover, 3D images are easily comprehensible which is important for communication between echocardiographers, interventional cardiologists and cardiac surgeons.

\section{Conflict of interest}

The authors state no conflict of interest.

\section{References}

[1] Vahanian A, Alfieri O, Andreotti F, Antunes MJ, Barón-Esquivias $\mathrm{G}$, Baumgartner $\mathrm{H}$, et al. Guidelines on the management of valvular heart disease. Joint Task Force on the Management of Valvular Heart Disease of the European Society of Cardiology (ESC) 1; European As- sociation for Cardio-Thoracic Surgery (EACTS). Eur Heart J 2012 Oct;33(19):2451-96.

[2] Von Ramm OT, Smith SW. Real time volumetric ultrasound imaging system. J Digit Imaging 1990;3:261-6.

[3] Sheikh K, Smith SW, von Ramm OT, Kisslo J. Real-time, three-dimensional echocardiography: feasibility and initial use. Echocardiography 1991;8:119-25.

[4] Yang HS, Bansal RC, Mookadam F. Practical guide for three-dimensional transthoracic echocardiography using a fully sampled matrix array transducer. Jam Soc Echocardiogr 2008;21:979-89.

[5] Flachskampf FA, Badano L, Daniel WG, Feneck RO, Fox KF, et al. Recommendations for transoesophageal echocardiography: update 2010. Eur J Echocardiogr 2010;11:557-76.

[6] Lang R, Badano L, Tsang W. EAE/ASE recommendations for image acquisition and display using three-dimensional echocardiography. J Am Soc Echocardiogr 2012;25:3-46.

[7] Salcedo E, Quaife R, Seres T, Carrol J. A framework for systematic characterization of the mitral valve by real time three dimensional transesopfageal echocardiography. J Am Soc Echocardiogr 2009;22:1087-99.

[8] Barlow JB. Perspectives on the mitral valve. Philadelphia, Pa: F.A. Davis; 1987.

[9] Van Mieghem N, Piazza N, Anderson R, Tzikas A, Nieman K, et al. Anatomy of the mitral valvular complex and its implications for transcatheter interventions for mitral regurgitation. J Am Coll Cardiol 2010;56:617-26.

[10] Carpentier A. Cardiac valve surgery: the 'French correction'. Journal of Thoracic and Cardiovascular Surgery 1983;86(3):323-37.

[11] Kumar N, Kumar M, Duran CMG. A revised terminologyfor recording surgical-findings of the mitral valve. Journal of Heart Valve Disease 1995;4(1):70-5.

[12] Silbiger JJ, Bazaz R. Contemporary insights into the functional anatomy of the mitral valve. Am Hear $J$ 2009;158(6):887-95.

[13] Nkomo VT, Gardin JM, Skelton TN, Gottdiener JS, et al. Burden of valvular heart disease: a population based study. Lancet 2006;368:1005-11.

[14] Lung B, Baron G, Messika-Zeitoun D, Detaint D, Vanoverschelde JL, et al. A prospective survey of patients with valvular heart disease in Europe: the Euro Heart Survey on Valvular Disease. Eur Heart J 2003;24:1231-43. 
[15] Chandra S, Salgo I, Sugeng L, Weinert L, Tsang W, et al. Characterization of degenerative mitral valve disease using morphologic analysis of real-time three-dimensional echocardiographic images: objective insight into complexity and planning of mitral valve repair. Circ Cardiovasc Imaging 2011;4:24-32.

[16] Filsoufi F, Carpentier A. Principles of reconstructive surgery in degenerative mitral valve disease. Semin Thorac Cardiovasc Surg 2007;19:103-10.

[17] Cotran RS, Kumar V, Collins T. Robbins pathologic base of disease. 6th ed. Philadelphia, Pa: W.B. Saunders Company; 1999. Chapter 13, p. 569-72.

[18] Faletra FF, de Castro S, Pandian NG. Atlas of real time 3D transesophageal echocardiography. Berlin: SpringerVerlag; 2010.

[19] Pan M, Medina A, Suarez de Lezo J, et al. Factors determining late success after mitral balloon valvulotomy. Am J Cardiol 1993;71(13):1181-5.

[20] Wilkins GT, Weyman AE, Abascal VM, et al. Percutaneous balloon dilatation of the mitral valve: an analysis of echocardiographic variables related to outcome and the mechanism of dilatation. Br Hear J 1988;60(4):299-308.

[21] Soliman OII, Anwar AM, Metawei AK, McGhie JS, Geleijnse ML, Ten Cate FJ. New scores for the assessment of mitral stenosis using real-time three-dimensional echocardiography. Curr Cardiovasc Imaging Rep 2011;4:370-7.

[22] Sutaria N, Shaw TR, Prendergast B, Northridge D. Transesophageal echocardiographic assessment of mitral valve commissural morphology predicts outcome after balloon mitral valvotomy. Heart 2006;92:52-7.

[23] Carpentier A, Adams D, Filsoufi F. Carpentier's reconstructive valve surgery. Philadelphia, Pa: Saunders; 2010. p. 116-8.
[24] Anwar A, et al. True mitral annulus diameter is underestimated by two dimensional echocardiography as evidenced by real-time three-dimensional echocardiography and magnetic resonance imaging. Int J Cardiovasc Imaging 2007;23:541-7.

[25] Ender J, Koncar-Zeh J, Mukherjee C, Jacobs S, Borger $\mathrm{MA}$, et al. Value ofaugmented reality-enhanced transesophageal echocardiography (TEE) for determining optimal annuloplasty ring sizeduring mitral valve repair. Ann Thorac Surg 2008;86:1473-8.

[26] Faletra FF, Moschovitis G, Auricchio A. Visualisation of thrombus formation on prosthetic valve by real-time three-dimensional transoesophageal echocardiography. Heart 2009;95:482.

[27] Kronzon I, Sugeng L, Perk G, Hirsh D, Weinert L, Garcia Fernandez MA, Lang RM. Real-time 3-dimensional transesophageal echocardiography in the evaluation of post-operative mitral annuloplasty ring and prosthetic valve dehiscence. J Am Coll Cardiol 2009;53:1543-7.

[28] Fischer G, Adams D. Real-time three-dimensional TEE guided repair of a paravalvular leak after mitral valve replacement. Eur J Echocardiogr 2008;9:868-9.

[29] Biner S, Rafique AM, Kar S, Siegel RJ. Live threedimensional transesophageal echocardiography-guided transcatheter closure of a mitral paraprosthetic leak by Amplatzer occluder. I Am Soc Echocardiogr 2008;21:1282.e7-9.

[30] Faletra F, et al. Role of real-time three dimensional transoesophageal echocardiography as guidance imaging modality during catheter based edge-to-edge mitral valve repair. Heart 2013;0:1-12.

[31] Colli A, et al. TEE-guided transapical beating-heart neochord implantation in mitral regurgitation. JACC Cardiovasc Imaging 2014;7(3):322-3. 